\title{
Soziologische Bildungsforschung
}

\author{
Claudia Diehl
}

\section{Rezension zu}

Rolf Becker/Heike Solga: Soziologische Bildungsforschung. Sonderheft 52 der Kölner Zeitschrift für Soziologie und Sozialpsychologie. Wiesbaden: Springer VS 2012. S. 498. ISBN-13: 978-3-658-00119-3. Preis: 49,95€

Das Sonderheft 52 der „Kölner Zeitschrift für Soziologie und Sozialpsychologie“ widmet sich der soziologischen Bildungsforschung und damit einem Thema, das in den vergangenen Jahren einen enormen Aufschwung erfahren hat. Angesichts der zahlreichen Wissenschaftlerinnen und Wissenschaftler, die sich mit dem Thema beschäftigen, der Fülle an Datensätzen, die zu seiner Untersuchung zur Verfügung stehen, und der Vielfalt der Themen, die in diesem Forschungsfeld bearbeitet werden, stellt die Auswahl der Einzelbeiträge eine beträchtliche Herausforderung bei der Herausgabe eines solchen Bandes dar. Das Auswahlproblem wurde für das vorliegende Sonderheft durch einen Call for Papers angegangen (S. 9). Die Herausgebenden argumentieren in ihrem Einleitungskapitel, dass die Art der eingesandten Manuskripte bereits viel über den aktuellen Zustand der deutschen Bildungsforschung aussagt. Unter anderem bescheinigen sie dieser gewisse „Verengung“ auf individuelle Bildungsentscheidungen, die unter gegebenen individuellen, familiären und institutionellen Rahmenbedingungen stattfinden. Es fehle hingegen an Studien, die diese Rahmenbedingungen - Kompetenzen, den institutionellen Aufbau des Schulsystems, die „,(meritokratische) Leistungsideologie“ (S. 16) - und ihre Folgen kritisch hinterfragen sowie konflikttheoretisch den „normativen und gesamtgesellschaftlichen Einbettungen des Bildungssystems“" nachgehen.

Dies freilich kann auch der vorliegende Band nur sehr ansatzweise leisten. Bei der Zusammenstellung der berücksichtigten Beiträge wird das Bemühen um inhaltliche und methodische Breite deutlich, es bestätigt sich aber auch die ebenfalls in der Einleitung konstatierte inhaltliche Orientierung vieler Studien an der Boudon'schen Unterschei-

Prof. Dr. C. Diehl $(\bowtie)$

Fachbereich Geschichte und Soziologie, Universität Konstanz,

Postfach 32, 78457 Konstanz, Deutschland

E-Mail: claudia.diehl@uni-konstanz.de 
dung von primären und sekundären Herkunftseffekten sowie die methodische Dominanz von Individualanalysen. Im Hinblick auf die verwendeten Datensätze ist die Breite der versammelten Manuskripte beeindruckend: SOEP, Mikrozensus, KOALA, PISA, ELEMENT, BIBB/BAuA Erwerbstätigenerhebung, der International Adult Literacy Survey der OECD, die Schweizer COCON Studie, der ALWA Datensatz des IAB, BiKS Daten, die HIS Studierendenberechtigtenbefragung, das ostmobil Jugendpanel, die Westdeutsche Lebensverlaufsstudie des MPI für Bildungsforschung sowie die Statistischen Jahrbücher der DDR. Ein Beitrag ist rein qualitativ angelegt (Pfahl), zwei weitere verwenden einen mixed-methods approach (Relikowski et al.; Groh-Samberg et al.), einem liegt eine Inhaltsanalyse europäischer Dokumente (Powell et al.) zugrunde. Die Studien beziehen sich ganz überwiegend auf Deutschland, wenige erstrecken sich auf mehrere Länderkontexte oder sind international vergleichend angelegt (Gottburgsen und Gross; Breen et al.; Solga; Groh-Samberg et al.), in einigen werden historische Entwicklungen nachgezeichnet (Jungbauer-Gans et al.; Breen et al.; Kleinert und Jacob sowie die Beiträge in Teil IV). Der Band ist in fünf Teile gegliedert. Diese widmen sich den Themen Bildungsungleichheit im Schulsystem (I.), Migration und Integration in das Bildungssystem (II.), Zugang zu Ausbildung und Studium und ihre Erträge (III.), Bildungserwerb im gesellschaftlichen Wandel (IV.) und gesellschaftliche Bildungskonzeption (V.).

Nach der programmatischen Einleitung beginnt Teil I mit der Behandlung einer vergleichsweise spezifischen Fragestellung, die für das Oberthema „Bildungsungleichheiten im Schulsystem“"von überschaubarer Relevanz ist. Wohlkinger und Ditton zeigen, dass bei einem Viertel der Familien auch am Ende der 4. Klasse die Übergangswünsche von Eltern und Kindern nicht übereinstimmen und dass sich bei dieser Konstellation keineswegs immer die Eltern durchsetzen, sondern die Aspirationen der Kinder einen eigenständigen Einfluss auf die Schulwahl haben. Jungbauer-Gans et al. geben einen lesenswerten Überblick über die Entwicklung des Privatschulbesuchs und zeigen, dass Privatschulen eher die Segregation als die Kompetenzen der Schülerinnen und Schüler fördern. Gottburgsen und Gross testen die Fruchtbarkeit des Intersektionalitätsparadigmas für die quantitative Forschung. Ihre empirischen Analysen bestätigen einmal mehr die Multidimensionalität von Bildungsungleichheiten. Allerdings konstatieren die Autorinnen, dass der Ansatz angesichts seiner „theoretischen Offenheit“ bei der Hypothesenformulierung wenig hilft. Da die Analysen zudem nur wenige signifikante und in die „richtige“ Richtung weisenden Interaktionseffekte liefern, fragt man sich nach der Lektüre, ob der Intersektionalitätsansatz wirklich einen sinnvollen Beitrag zu einer theoriegeleiteten empirischen Bildungsforschung leistet.

Im Zentrum des zweiten Teils des Bandes steht das Thema „Migration und Integration in das Bildungssystem“ und hier zeigt sich einmal mehr, dass der Migrationshintergrund an sich keine Barriere für Bildungserfolg darstellt. Relikowski et al. greifen das Thema der hohen Bildungsaspirationen von Migranten auf und berücksichtigen dabei den Zugang zu tertiärer Bildung im Herkunftsland. Sie können empirisch belegen, dass gerade Einwanderern aus Ländern mit beschränkten Aufstiegsopportunitäten im Zielland offenbar viel daran liegt, dass ihre Kinder verpasste Chancen nachholen. Becker und Beck bestätigen in ihrer Analyse von ELEMENT Daten, dass Migrantenkinder bei gleichem Abschneiden in Kompetenzmessungen bei der Notengebung nicht benachteiligt werden (die Koeffizienten für die soziale Herkunft sind bei den Mathematiknoten offenbar falsch) und eher 
häufiger als seltener aufs Gymnasium wechseln sowie eine entsprechende Empfehlung erhalten. Allerdings fallen hier die deutlich negativen, wenngleich nicht signifikanten Effekte für die Türken und „Araber“ auf. Unklar bleibt, warum von vorneherein von statistischer Diskriminierung als mögliche Ursache ethniespezifischer Beurteilungsstrategien ausgegangen wird. Söhns Analyse zur rechtlichen Stratifikation greift einen wichtigen und vernachlässigten Aspekt in der Forschung zu ethnischen Bildungsungleichheiten auf, verdeutlicht aber auch die Grenzen, an die Analysen mit dem Mikrozensus häufig stoßen: den Rechtsstatus über die Herkunftsländer zu operationalisieren und Resteffekte der Herkunft als Einfluss der rechtlichen Rahmenbedingungen zu deuten, bleibt im Sinne einer Kausalerklärung unbefriedigend, v. a. weil nur bei Einwanderern aus der „ExUdSSR “ und Polen eine Unterscheidung zwischen dem Einreisestatus als Deutscher oder Ausländer möglich ist.

Im dritten Teil zu „Zugang zu Ausbildung und Studium und ihre Erträge“ gehen Kleinert und Jacob der ungleichheitsrelevanten Frage nach, wie sich die Chancen von Absolventen höherer oder niedrigerer Schulabschlüsse, eine berufliche Ausbildung aufnehmen zu können, im Zeitverlauf entwickelt haben. Die Chancen ersterer sind weitgehend stabil geblieben und recht unbeeinflusst von Konjunkturzyklen und Arbeitslosenquoten, während die benachteiligter Schülerinnen und Schüler sich nicht nur im Zeitverlauf verschlechtert haben, sondern ihre Übergangschancen stärker Marktschwankungen unterliegen. Buchmann und Kriesi zeigen anhand von Längsschnittdaten, dass die Wahl von Männer- und Frauenberufen von den elterlichen Fähigkeitszuschreibungen und der Unterstützung für einen geschlechtsuntypischen Beruf beeinflusst wird, obwohl diese Zuschreibungen offenbar eher stereotypenbasiert sind und in keinem Zusammenhang mit den gemessenen Kompetenzen stehen. Auch horizontale und vertikale Herkunftseffekte bei der Entscheidung für ein Hochschulstudium und die Studienfachwahl werden in diesem Teil des Sonderhefts behandelt (Lörz). Hall untersucht, ob schulische Ausbildungen auch dann niedrigere Bildungsrenditen zeigen als betriebliche, wenn bei den entsprechenden Analysen nach Geschlecht differenziert wird. Dies ist den sehr klaren Analysen zufolge nicht der Fall, was auch viel damit zu tun hat, dass die typischen dualen Frauenberufe häufig im Niedriglohnsegment angesiedelt sind.

Im Abschnitt „Bildungserwerb im gesellschaftlichen Wandel“ sticht besonders der Beitrag von Breen et al. ins Auge, die eine vergleichsweise groß angelegte - und teilweise bereits an anderer Stelle publizierte - Analyse zu der Frage präsentieren, wie sich der Einfluss von Herkunft und Geschlecht auf Bildungsdisparitäten im Zeitverlauf verändert hat. Sie widerlegen ältere Befunde, denen zufolge Herkunftseffekte weitgehend stabil geblieben sind und zeigen, dass die entsprechenden Analysen offenbar auf zu kleinen Fallzahlen basierten. Zusätzlich wird dargestellt, dass sich dieser Trend in abgeschwächter Form in Deutschland auch in jüngeren Jahren fortgesetzt hat. Ergänzt werden die deskriptiven Analysen um eine lesenswerte theoretische Diskussion der Wirkmechanismen, die hinter den innerhalb von Familien erzeugten Geschlechterdisparitäten und den zwischen Familien entstehenden Herkunftseffekten stehen. Es folgen zwei Beiträge zum Thema Geschlechterungleichheiten. Reisz et al. analysieren die Studienfachwahl in der DDR und der BRD, Helbigs Explanandum ist die Umkehrung der Geschlechterverhältnissen beim Erwerb des Abiturs. Anhand von Analysen amtlicher Daten zeigt der Autor, dass dafür Änderungen im Übergangsverhalten und den schulischen Verläufen, nicht aber 
in den Kompetenzen eine Rolle gespielt haben - ein erneuter Hinweis auf die Bedeutung sekundärer Effekte.

Der letzte Teil des Bandes wird von Pfahl, Powell et al. und Solga bestritten und behandelt unter dem Stichwort „Gesellschaftliche Bildungskonzeptionen“ Themen und methodische Zugangswege, die in der soziologischen Bildungsforschung eher am Rande vorkommen. Die Studie von Pfahl illustriert die Stigmatisierung von Sonderschülern anhand einer qualitativen Studie, Powell et al. analysieren inhaltsanalytisch, ob die Neuordnung der europäischen Berufs- und Hochschulbildung eher amerikanischen oder europäischen Vorbildern folgt und zeigen, dass hier offenbar kein einzelnes Modell Pate gestanden, sondern sich eine Vielzahl von Einflüssen niedergeschlagen hat. Solga beschließt den Band, indem sie den Zusammenhang zwischen Chancengleichheit, Bildungsergebnisungleichheit - v. a. im Sinne von Bildungsarmut - und Einkommensungleichheit anhand eines europäischen Vergleichs von PISA Daten untersucht. Der Einfluss der Chancengleichheit im Bildungssystem darf ihren Ergebnissen zufolge nicht unterschätzt werden, letztlich bleibe Umverteilung der wichtigste Mechanismus zur Reduktion von Einkommensungleichheiten.

Nach dem Lesen des Rezensionsexemplars - das für die Lektüre ,in einem Schwung“ steht, die für Sonderbände sicherlich ungewöhnlich ist - hat man einen interessanten und vielfältigen Einblick in die aktuelle deutsche Bildungsforschung erhalten. Mehrere Beiträge zeigen, wie fruchtbar der Versuch ist, Einblick in allgemeinere theoretische Mechanismen durch internationale Vergleiche und die Betrachtung historischer Verläufe zu erhalten. Man kann die in der Einleitung beschriebene Diagnose einer Verengung der Bildungsforschung nachvollziehen, fragt sich aber bisweilen, ob diese tatsächlich der Fokussierung auf die Boudon'sche Individualperspektive geschuldet ist. Eine kritischere Bildungsforschung braucht ja zuvörderst empirische Evidenzen für die Wirkweise der Institutionen, Praktiken und Regelungen, die zu unerwünschten Effekten führen, zunächst einmal unabhängig davon, ob man diese nun in anhaltenden Herkunftsdisparitäten, in fortbestehender Bildungsarmut oder in der frühen Stigmatisierung derer sieht, denen die „Passung“ ins Bildungssystem scheinbar fehlt. Vor diesem Hintergrund hätte man sich statt der Behandlung einiger doch sehr spezifischer Detailfragen etwas mehr Einblick in die neuere Forschung zu den Auswirkungen und der Bedeutung gerade schulischer Strukturen und institutioneller Praktiken gewünscht, etwa zur Segregation in Schulklassen, zu Regelungen auf Länderebene wie etwa der Verbindlichkeit von Bildungsempfehlungen oder der Dauer der Primarstufe oder auch zu informellen Praktiken wie etwa dem Umgang der Lehrenden mit vermeintlich „fehlenden“ sprachlichen oder kulturellen Ressourcen.

Kritisch angemerkt sei auch, dass die Anordnung der Einzelbeiträge und ihre thematische Zuordnung an manchen Stellen etwas beliebig wirkt. So bietet der Band einen guten Überblick über neuere Forschungen und Befunde zum Thema Geschlechterungleichheiten. Leider sind diese über den Band verteilt und wurden nicht, ähnlich wie - oder sogar anstatt - der Beiträgen zu ethnischen Effekten, in einem eigenen Teil zusammengefasst und damit stärker herausgestellt. Erwähnt sei auch, dass es dem Band an einigen Stellen an editorischer Sorgfalt mangelt. Offensichtlich falsche Koeffizienten, Tabellen- und Abbildungsbeschriftungen und Schreibfehler hätten in einem gründlichen Begutachtungsverfahren eigentlich auffallen müssen. 